\title{
An inverse method for radio frequency phased array coil design in magnetic resonance imaging equipment
}

Peter T. While ${ }^{1} \quad$ Larry K. Forbes ${ }^{2} \quad$ Stuart Crozier $^{3}$

(Received 27 July 2007; revised 23 August 2007)

\begin{abstract}
Magnetic resonance imaging is a popular non-invasive technique for investigating soft tissue structures within the human body. Many design methods now exist for their principle hardware components, such as the radio frequency (RF) coils. A popular RF coil type is the RF phased array, comprised of many closely spaced coils covering a large volume. A time harmonic inverse method is presented for the theoretical design of RF phased arrays. The method allows any array size to be considered where the focus is on optimal coil geometry and position of individual coils. An ill-conditioned integral equation is solved using a regularisation strategy in which the error between
\end{abstract}

See http://anziamj.austms.org.au/ojs/index.php/ANZIAMJ/article/view/334 for this article, (C) Austral. Mathematical Soc. 2007. Published August 31, 2007. ISSN $1446-8735$ 
induced and target magnetic fields is minimised along with an additional constraint related to the curvature of the coil windings. The method is demonstrated for a number of design considerations and includes the ability to focus the RF field to arbitrary locations within the coil volume. The effect of the choice of magnetic field polarisation direction is also investigated using the model.

\section{Introduction}

Magnetic resonance imaging (MRI) scanners use a combination of three main hardware components to acquire an image: a primary magnet, gradient coils, and radio frequency (RF) coils [5, p.137, e.g.]. RF coils have two modes of operation: transmit and receive. In transmit mode they must induce a homogeneous magnetic field (perpendicular to the primary field) such that all locations within the sample to be imaged experience an equal weighting of RF energy. In receive mode they must pick up signals from all locations with an equal gain and achieve this with a high signal-to-noise ratio (SNR). RF phased arrays are comprised of many closely spaced coils covering a large volume. This affords high SNR and resolution over a large field of view, yet with no increase in imaging time [9]. In RF phased array coil design, it is typical to take established coil structures, such as simple loops, and optimise the amplitudes and phases of the drives of each coil [7, e.g.]. Other methods concentrate more on optimising with respect to coil placement $[4$, e.g.].

The importance of coil geometry in obtaining optimal image quality and SNR is stressed by Sodickson et al. [10], who also suggest a possible role for the target field method in coil array design. The target field method is well established in gradient coil design [11]. It is an inverse method whereby one specifies a desired magnetic field within some region of interest (the DSV) and then seeks an appropriate coil current density solution. Forbes and Crozier [3] extended the method using a Fourier series representation for the current density such that finite length coils are considered without ap- 
proximation. They used a regularisation strategy to solve an ill-conditioned integral equation.

The design of high frequency RF coils is considered by Lawrence et al. [6], who use a full wave approach involving time harmonic Green's functions to obtain induced field expressions. While et al. [12] extended this work by considering a more general current density and a regularisation strategy that allows a trade-off between field accuracy and coil simplicity. The important cases of RF shielding and patient loading are considered by While et al. [13, 14]. The method is adapted to the design of RF phased array coils by While et al. [15]. The current article presents new results using this adapted method, which is revisited briefly in the following section. Example coil winding solutions and their corresponding induced fields are discussed in Section 3. A variety of phased array design considerations are investigated, including array size, axial and radial asymmetry in DSV location (field focussing) and field polarisation.

\section{Method}

In this section, the governing integral equation for the magnetic field, the chosen form of the current density, and the regularisation process, are presented. The geometry of the problem is shown in Figure 1. On a cylinder of length $2 L$ and radius $a$ there exists an unknown current density vector $\mathbf{j}\left(\theta^{\prime}, z^{\prime}\right)$, for which we intend to solve, such that it induces some desired magnetic field upon the surface of an interior target region (DSV). This target region is chosen to be a sphere of radius $c$, centred at the point $(r, \theta, z)=\left(r_{c}, \theta_{c}, z_{c}\right)$.

A time harmonic analysis is considered involving sinusoidal time dependence of the form $e^{i \omega t}$. By introducing vector and scalar potentials along with the Lorentz gauge, Helmholtz equations can be obtained from Maxwell's reduced equations [8, p.259, e.g.]. Free space Green's functions solve these equations and yield an integral expression for the magnetic induction vec- 


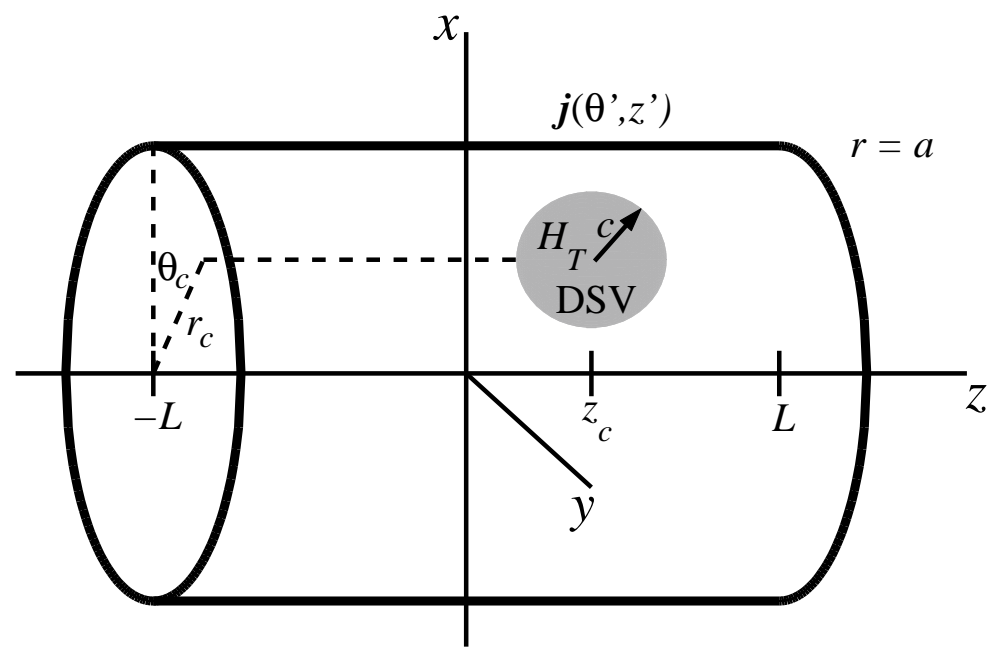

Figure 1: The model used to describe an RF head coil and an arbitrarily positioned spherical target region (DSV).

tor [15]. Expanding this expression in terms of cylindrical polar coordinates, we obtain the following expressions for the $x$ - and $y$-components of the induced magnetic field vector at any field point $(r, \theta, z)$ :

$$
\begin{aligned}
\bar{H}_{x}(r, \theta, z)= & \frac{a}{2 \pi} \int_{0}^{2 \pi} \int_{-L}^{L}\left[\left(a \sin \theta^{\prime}-r \sin \theta\right) \bar{j}_{z}\left(\theta^{\prime}, z^{\prime}\right)\right. \\
& \left.-\left(z^{\prime}-z\right) \cos \theta^{\prime} \bar{j}_{\theta}\left(\theta^{\prime}, z^{\prime}\right)\right] \times e^{-i \alpha R}\left(\frac{i \alpha}{R^{2}}+\frac{1}{R^{3}}\right) d z^{\prime} d \theta^{\prime}, \\
\bar{H}_{y}(r, \theta, z)= & -\frac{a}{2 \pi} \int_{0}^{2 \pi} \int_{-L}^{L}\left[\left(a \cos \theta^{\prime}-r \cos \theta\right) \bar{j}_{z}\left(\theta^{\prime}, z^{\prime}\right)\right. \\
& \left.+\left(z^{\prime}-z\right) \sin \theta^{\prime} \bar{j}_{\theta}\left(\theta^{\prime}, z^{\prime}\right)\right] \times e^{-i \alpha R}\left(\frac{i \alpha}{R^{2}}+\frac{1}{R^{3}}\right) d z^{\prime} d \theta^{\prime}
\end{aligned}
$$

where

$$
R=\left[a^{2}+r^{2}-2 a r \cos \left(\theta^{\prime}-\theta\right)+\left(z^{\prime}-z\right)^{2}\right]^{1 / 2} .
$$


and $\alpha=\omega \sqrt{\mu_{0} \epsilon_{0}}$. We wish to solve for the current density components $j_{\theta}\left(\theta^{\prime}, z^{\prime}\right)$ and $j_{z}\left(\theta^{\prime}, z^{\prime}\right)$, which makes equations (1) and (2) Fredholm integral equations of the first kind [2, p.299, e.g.]. Solutions are highly ill-conditioned and a regularisation strategy overcomes this problem.

The streamfunction method is used to obtain coil windings, which demands a divergence free current density [1, e.g.]. The coil cylinder is divided into $K \times Q$ subregions in $\theta$ and $z$. The current densities in each subregion must be independent of one another and the azimuthal and axial current density components must fall to zero at each subregion boundary in $\theta$ and $z$, respectively. Appropriate forms for the $(k, q)$ subregion are therefore

$$
\begin{aligned}
& \bar{j}_{z}^{k q}\left(\theta^{\prime}, z^{\prime}\right)=-\sum_{n=1}^{N} \sum_{m=1}^{M} \frac{m K}{2 a} c_{m n}^{k q} \sin \left(\frac{n Q \pi\left(z^{\prime}+L\right)}{2 L}\right) \cos \left(\frac{m K \theta^{\prime}}{2}\right), \\
& \bar{j}_{\theta}^{k q}\left(\theta^{\prime}, z^{\prime}\right)=\sum_{n=1}^{N} \sum_{m=1}^{M} \frac{n Q \pi}{2 L} c_{m n}^{k q} \cos \left(\frac{n Q \pi\left(z^{\prime}+L\right)}{2 L}\right) \sin \left(\frac{m K \theta^{\prime}}{2}\right),
\end{aligned}
$$

with the corresponding streamfunction

$$
\bar{\psi}^{k q}\left(\theta^{\prime}, z^{\prime}\right)=\sum_{n=1}^{N} \sum_{m=1}^{M} c_{m n}^{k q} \sin \left(\frac{n Q \pi\left(z^{\prime}+L\right)}{2 L}\right) \sin \left(\frac{m K \theta^{\prime}}{2}\right),
$$

and with $M$ and $N$ sufficiently large. Equations (4)-(6) exist only for $-L+$ $(q-1) \frac{2 L}{Q} \leq z^{\prime} \leq-L+q \frac{2 L}{Q}$ and $(k-1) \frac{2 \pi}{K} \leq \theta^{\prime} \leq k \frac{2 \pi}{K}$, where $k=1: K$ and $q=1: Q$. We intend to solve for the coefficients $c_{m n}^{k q}(m=1: M, n=1: N$, $k=1: K, q=1: Q)$ to find the current density over the entire cylinder.

We minimise the error $\Phi$ between induced and desired 'target' magnetic fields over the surface of the DSV. This error is

$$
\Phi=\oiint\left\{\left[\bar{H}_{x}-\bar{H}_{T x}\right]^{2}+\left[\bar{H}_{y}-\bar{H}_{T y}\right]^{2}\right\} d S,
$$

where $\bar{H}_{T x}$ and $\bar{H}_{T y}$ are the homogeneous target field components. Specification of limits and surface element for the surface integral depends on the 
location of the DSV. Consider the case where the DSV is symmetric about the $z$-axis but centred at some axially asymmetric point $(r, \theta, z)=\left(0,0, z_{c}\right)$. Equations (4) and (5) are firstly substituted into equations (1) and (2). These equations are then substituted into equation (7), which is minimised with respect to the unknown coefficients $c_{m n}^{k q}$. This yields the condition

$$
\begin{aligned}
\frac{\partial \Phi}{\partial c_{s t}^{f g}}= & -2 c \int_{-c+z_{c}}^{c+z_{c}} \int_{-\pi}^{\pi}\left[\bar{H}_{T x} U_{s t}^{f g}\left(r_{1}, \theta, z\right)+\bar{H}_{T y} V_{s t}^{f g}\left(r_{1}, \theta, z\right)\right] d \theta d z \\
& +2 c \sum_{n=1}^{N} \sum_{m=1}^{M} \sum_{q=1}^{Q} \sum_{k=1}^{K} c_{m n}^{k q} \int_{-c+z_{c}}^{c+z_{c}} \int_{-\pi}^{\pi}\left[U_{m n}^{k q}\left(r_{1}, \theta, z\right) U_{s t}^{f g}\left(r_{1}, \theta, z\right)\right. \\
& \left.+V_{m n}^{k q}\left(r_{1}, \theta, z\right) V_{s t}^{f g}\left(r_{1}, \theta, z\right)\right] d \theta d z=0
\end{aligned}
$$

where

$$
r_{1}(z)=\sqrt{c^{2}-\left(z-z_{c}\right)^{2}},
$$

and $U_{m n}^{k q}$ and $V_{m n}^{k q}$ represent double integrals in $\theta$ and $z$ [15]. However, we also wish to consider a DSV centred about some arbitrarily located point $(r, \theta, z)=$ $\left(r_{c}, \theta_{c}, z_{c}\right)$. This requires a lengthy geometric argument and a change of variables to ensure convergence when numerically integrating in this radially asymmetric case. While et al. [15] describe the resulting condition.

The integrals in equation (8) in terms of $\theta$ and $z$ are evaluated numerically using the Trapezoidal rule. A system of linear equations results in terms of the coefficients $c_{m n}^{k q}$ and is expressed in matrix form. However, the ill-conditioned nature of this problem leads to errors in the corresponding solution and so a regularisation strategy is implemented [2, p.307, e.g.]. The function $\Phi$ is replaced by a residual error $R$ by adding a weighting $\lambda$ (the regularising parameter) of a penalty function $\Pi$, which represents some constraint on the current density. The choice of penalty function is left to the designer and in this article we consider a 'smoothness' penalty function, which minimises the curvature of the coil windings. For the current density components given by equations (4) and (5), the smoothness condition 
becomes

$$
\frac{\partial \Pi}{\partial c_{s t}^{f g}}=\frac{2 a \pi L}{K Q}\left[\left(\frac{s K}{2 a}\right)^{2}+\left(\frac{t Q \pi}{2 L}\right)^{2}\right]^{3} c_{s t}^{f g} .
$$

Further details of this regularisation strategy are described by While et al. [15]. The conditioning of the matrix equation can now be improved by increasing the value of the regularising parameter $\lambda$. A trade-off exists between having a well-conditioned system, for which $\lambda$ is large, and being able to match the target field accurately, for which $\lambda$ must be small [3]. To obtain fixed winding patterns, we must contour the time average of the streamfunction $\bar{\psi}\left(\theta^{\prime}, z^{\prime}\right)$ in equation (6). The fields induced by these coil windings are plotted using equations (1) and (2) to test the accuracy of the final solution.

\section{$3 \quad$ Results}

In this section, coil winding solutions for a variety of phased array cases are displayed and discussed, both in terms of their geometric shape and the homogeneity of their correspondingly induced magnetic fields. Dimensions represent a head coil of length $2 L=0.3 \mathrm{~m}$ and radius $a=0.125 \mathrm{~m}$ (see Figure 1). We firstly consider an axially asymmetrically located target region of radius $c=0.05 \mathrm{~m}$, centred about the point $(r, \theta, z)=(0,0,0.05)$. The $x$ and $y$-components of the target magnetic field represent a homogeneous $90^{\circ}$ linearly polarised field (y-polarisation) by setting $\bar{H}_{T x}(r, \theta, z)=0 \mathrm{~A} / \mathrm{m}$ and $\bar{H}_{T y}(r, \theta, z)=1 \mathrm{~A} / \mathrm{m}$. The frequency of operation was chosen to be $190 \mathrm{MHz}$ representing high field imaging $(4.5 \mathrm{~T})$. The program MATLAB ${ }^{\mathrm{TM}}$ was used extensively for numerical integration, solving matrix equations and plotting figures of interest. Note the further examples and subsequent discussion by While et al. [15].

We initially consider a $K \times Q=4 \times 2$ array. Regularising using the smoothness penalty function reduces the condition number of the matrix system from the order of $10^{21}$ to a minimum of $10^{4}$ when $\lambda=10^{-13}$. Figure 2 


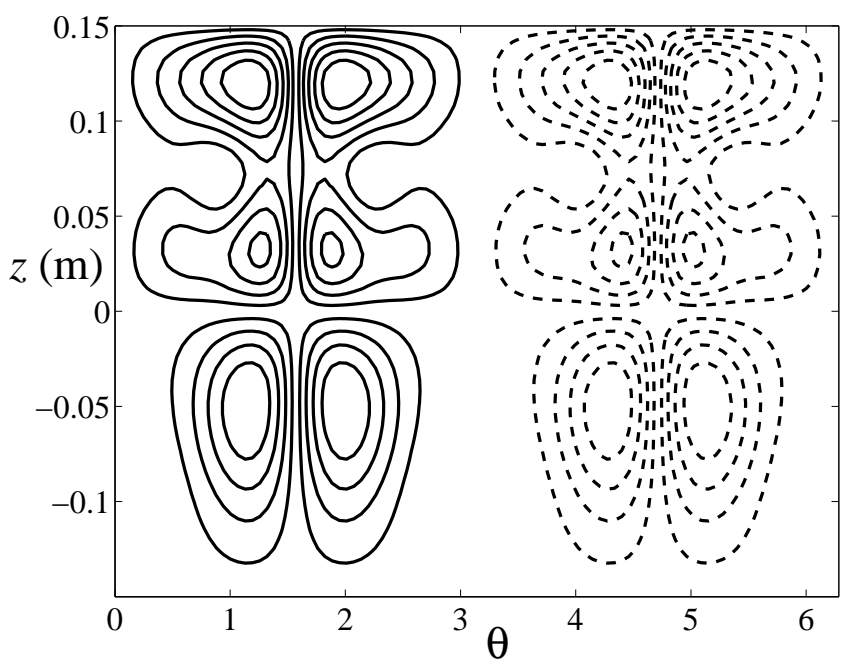

FiguRE 2: Coil windings for a $4 \times 2$ array with an axially asymmetrically located DSV.

displays the corresponding coil winding pattern. The dashed lines indicate that the current is reversed in those portions of the coil. Note the axial asymmetry of the coil array. Figure 3 displays the induced $\bar{H}_{y}$ field at the coil centre along a line of constant $r=0 \mathrm{~m}$. The vertical dashed lines indicate the boundary of the DSV and the horizontal dashed line indicates the desired target magnetic field. The induced field matches the target field to a good level of accuracy for most of the DSV, despite the extreme level of axial asymmetry, but falls below the value of $1 \mathrm{~A} / \mathrm{m}$ towards the end of the coils. Using a value of $\lambda$ smaller than $10^{-13}$ results in more complex windings but a more desirable field, and vice versa for a larger value of $\lambda$.

Figure 4 displays the coil winding solution for a $6 \times 3$ array, obtained by altering the values of $K$ and $Q$ in equations (8) and (9). These coil windings are simpler than those of the $4 \times 2$ array of Figure 2. The corresponding induced $\bar{H}_{y}$ field for the $6 \times 3$ array displays a superior level of homogeneity when compared to the $4 \times 2$ array. That is, increasing array size for this axially 


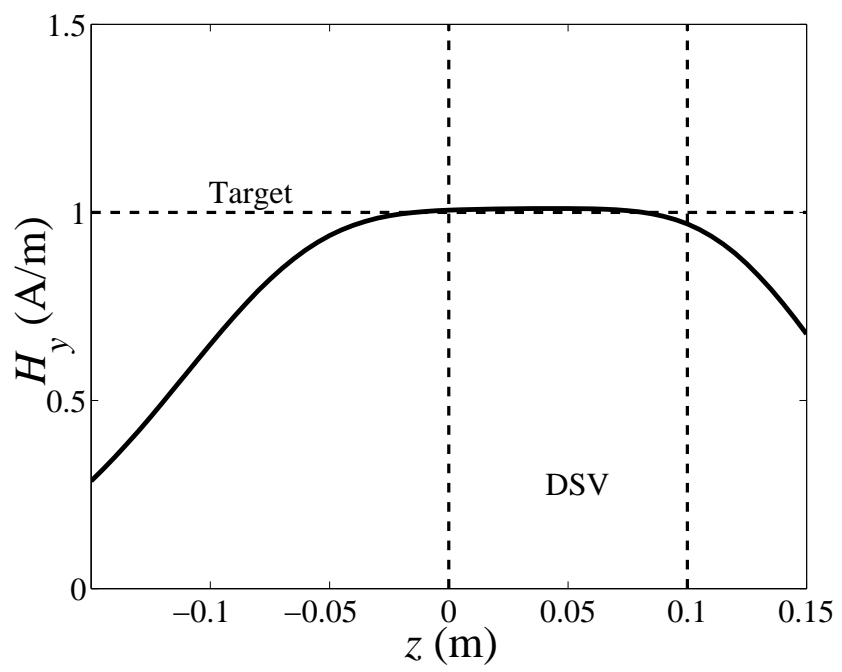

FiguRe 3: Induced $\bar{H}_{y}(r, \theta, z)$ field along coil centre corresponding to the $4 \times 2$ array of Figure 2 .

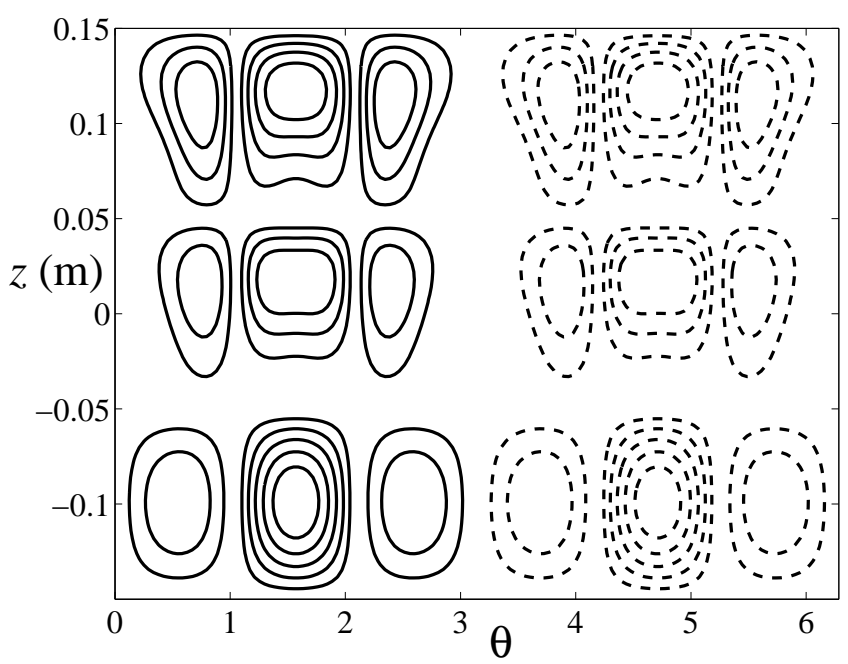

Figure 4: Coil windings for a $6 \times 3$ array with an axially asymmetrically located DSV. 


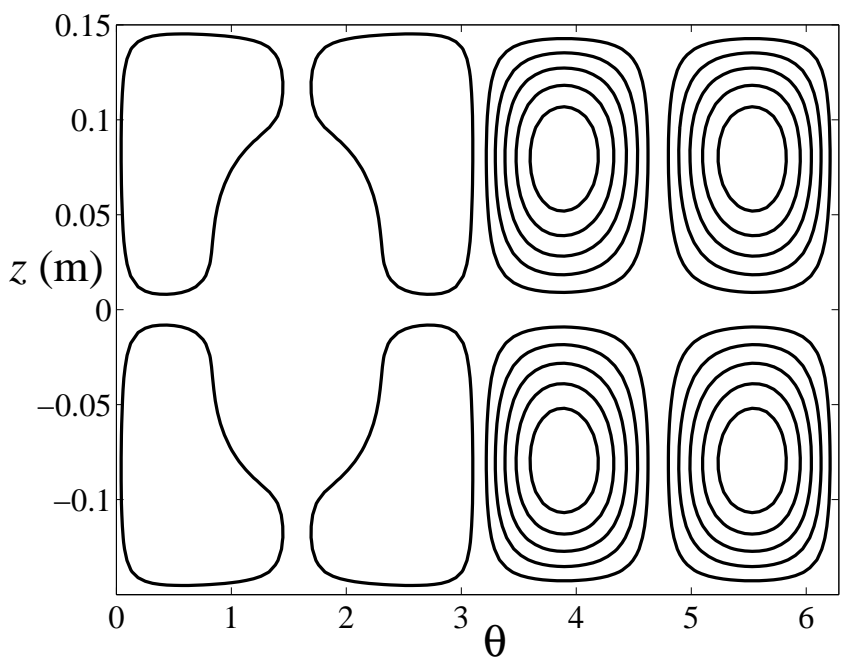

FIGURE 5: Coil windings for a $4 \times 2$ array with a radially asymmetrically located DSV, assuming a $y$-polarised field.

asymmetric case leads to both simpler winding patterns and improved field homogeneity, at the cost of additional circuitry.

The more complex case of radial asymmetry was also considered. The DSV was chosen to have radius $c=0.025 \mathrm{~m}$ and centred about some point $\left(r_{c}, \theta_{c}, z_{c}\right)=(0.05, \pi / 2,0)$ off axis. Applications for a head coil exist in focussing the field to a particular location in the brain, for example. For a radially asymmetrically located DSV, the direction of linear polarisation of the target field becomes important. Two types of linear polarisation were considered: $y$-polarisation $\left(90^{\circ}\right)$ and $x$-polarisation $\left(0^{\circ}\right)$. The former represents polarisation in the same direction as that in which the DSV is offset from the $z$-axis, and the latter represents a perpendicular direction.

Figure 5 displays the coil winding solution for a $4 \times 2$ array assuming a $y$-polarised target field $\left(\lambda=10^{-13}\right)$. Notice that we obtain large current on the half of the coil cylinder that lies furthest away from the DSV, and small 


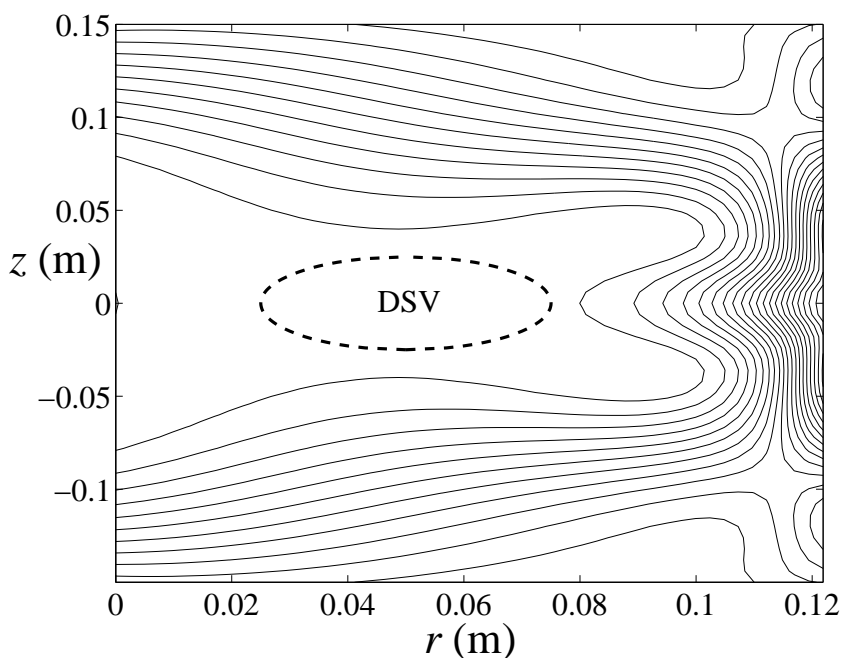

FiguRE 6: Induced $\bar{H}_{y}(r, \theta, z)$ field corresponding to the $4 \times 2$ array of Figure 5 , displayed as a contour plot $(5 \%)$ on the $(r, z)$ plane $\theta=\pi / 2$.

current on the half closest to the DSV. Figure 6 shows the correspondingly induced $\bar{H}_{y}$ field as a $5 \%$ contour plot on the $(r, z)$ plane at $\theta=\pi / 2$. The curved dashed line shows the boundary of the DSV in this plane, within which the field is found to be homogeneous. As for the axially asymmetric case, increasing the array size leads to improved field homogeneity.

Figure 7 displays the coil winding solution for a $4 \times 2$ array, assuming an $x$-polarised, radially asymmetrically located target field $\left(\lambda=10^{-13}\right)$. This array is of a significantly different form to that for the $y$-polarisation case of Figure 5, in terms of coil shape, current amplitude and phase. The windings for the $x$-polarised case are placed much closer to the DSV than in the $y$-polarised case, which would result in higher efficiency and sensitivity for these coil designs. In addition, the induced $\bar{H}_{y}$ field displays a superior level of homogeneity within the DSV for the $x$-polarised case. This improvement in efficiency, sensitivity and field homogeneity illustrates the importance of polarisation considerations in obtaining phased array coil windings for radially 


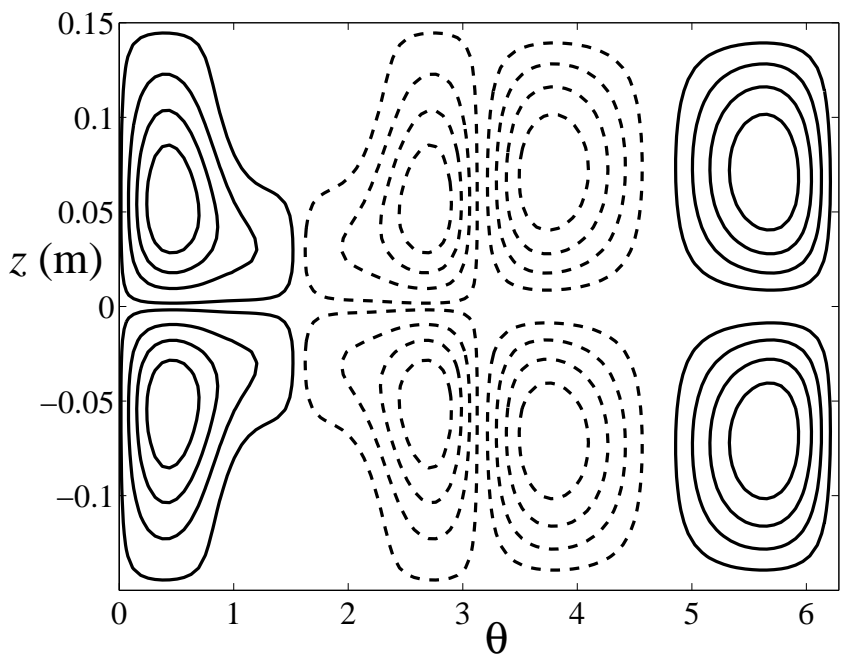

Figure 7: Coil windings for a $4 \times 2$ array with a radially asymmetrically located DSV, assuming an $x$-polarised field.

asymmetrically located target regions.

\section{Conclusion}

An inverse method for high frequency RF phased array coil design has been presented. This method involved the minimisation of the error between induced and target magnetic fields over the surface of the DSV. In considering a phased array coil, the coil cylinder was divided into a set of subregions and the current density in each represented using Fourier series expansions. The resulting ill-conditioned integral equation was solved using regularisation, whereby an additional constraint was imposed on the current density in terms of minimum curvature of the coil windings. This allowed a trade-off to be realised between coil array simplicity and magnetic field homogeneity. 
The ability to focus the RF field was incorporated into the model by varying the location of the DSV before solving for the current density coefficients. Both axial and radial asymmetry were investigated for a number of array sizes and field polarisations. For the case of axial asymmetry, in which the DSV was placed near one end of the coil, winding pattern solutions were obtained for $4 \times 2$ and $6 \times 3$ arrays that were capable of inducing homogeneous fields within the DSV. Increasing array size in this case led to simpler coil windings and improved field homogeneity. For the case of radial asymmetry, in which the DSV was placed closer to one side of the coil, the choice of the direction of magnetic field polarisation had a considerable effect on the form of the winding pattern solution. The important effects of coil coupling and sample loading may be incorporated into the model in future work.

\section{References}

[1] Brideson M. A., Forbes L. K. and Crozier S. 2002. Determining complicated winding patterns for shim coils using streamfunctions and the target-field method, Concepts in Magnetic Resonance 14(1), 9-18. doi:10.1002/cmr.10000 C5

[2] Delves L. M. and Mohamed J. L. 1985. Computational methods for integral equations, Cambridge: Cambridge University Press. C5, C6

[3] Forbes L. K. and Crozier S. 2002. A novel target-field method for finite-length magnetic resonance shim coils: II. tesseral shims, Journal of Physics D: Applied Physics 35, 839-849. doi:10.1088/0022-3727/35/9/303 C2, C7

[4] Hardy C. J., Cline H. E., Giaquinto R. O., Niendorf T., Grant A. K. and Sodickson D. K. 2006. 32-element receiver-coil array for cardiac imaging, Magnetic Resonance in Medicine 55, 1142-1149. doi:10.1002/mrm.20870 C2 
[5] Jin J. 1999. Electromagnetic Analysis and Design in Magnetic Resonance Imaging, Florida: CRC Press. C2

[6] Lawrence B. G., Crozier S., Yau D. D. and Doddrell D. M. 2002. A time-harmonic inverse methodology for the design of RF coils in MRI, IEEE Transactions on Biomedical Engineering 49(1), 64-71. doi:10.1109/10.972841 C3

[7] Li B. K., Liu F. and Crozier S. 2005. Focused, eight-element transceive phased array coil for parallel magnetic resonance imaging of the chest - theoretical considerations, Magnetic Resonance in Medicine 53, 1251-1257. doi:10.1002/mrm.20505 C2

[8] Ramo S., Whinnery J. R. and van Duzer T. 1967. Fields and waves in communication electronics, New York: John Wiley and Sons, Inc. C3

[9] Roemer P. B., Edelstein W. A., Hayes C. E., Souza S. P. and Mueller O. M. 1990. The NMR phased array, Magnetic Resonance in Medicine 16(2), 192-225. doi:10.1002/mrm.1910160203 C2

[10] Sodickson D. K., McKenzie C. A., Ohliger M. A., Yeh E. N. and Price M. D. 2002. Recent advances in image reconstruction, coil sensitivity calibration, and coil array design for SMASH and generalized parallel MRI, Magnetic Resonance Materials in Physics, Biology and Medicine 13, 158-163. C2

[11] Turner R. 1986. A target field approach to optimal coil design, Journal of Physics D: Applied Physics 19, L147-L151.

doi:10.1088/0022-3727/19/8/001 C2

[12] While P. T., Forbes L. K. and Crozier S. 2005a. A time-harmonic target-field method for designing unshielded RF coils in MRI, Measurement Science and Technology 16, 997-1006. doi:10.1088/0957-0233/16/4/012 C3 
[13] While P. T., Forbes L. K. and Crozier S. 2005b. A time-harmonic target-field method for designing shielded RF coils in MRI, Measurement Science and Technology 16, 1381-1393. doi:10.1088/0957-0233/16/6/021 C3

[14] While P. T., Forbes L. K. and Crozier S. 2006. An inverse method for designing loaded RF coils in MRI, Measurement Science and Technology 17, 2506-2518. doi:10.1088/0957-0233/17/9/019 C3

[15] While P. T., Forbes L. K. and Crozier S. 2007. An inverse method for designing RF phased array coils in MRI - theoretical considerations, Measurement Science and Technology 18, 245-259. doi:10.1088/0957-0233/18/1/031 C3, C4, C6, C7 


\section{Author addresses}

1. Peter T. While, School of Maths \& Physics, University of Tasmania, Private Bag 37, Hobart, Tasmania 7001, Australia. mailto:pwhile@utas.edu.au

2. Larry K. Forbes, School of Maths \& Physics, University of Tasmania, Private Bag 37, Hobart, Tasmania 7001, Australia.

3. Stuart Crozier, School of Information Technology \& Electrical Engineering, University of Queensland, St. Lucia, Brisbane, Queensland 4072, Australia. 